\title{
Uptake of ricinB-quantum dot nanoparticles by a macropinocytosis-like mechanism
}

Tore Geir Iversen ${ }^{1,2^{*}}$, Nadine Frerker ${ }^{1,2}$ and Kirsten Sandvig ${ }^{1,2,3}$

\begin{abstract}
Background: There is a huge effort in developing ligand-mediated targeting of nanoparticles to diseased cells and tissue. The plant toxin ricin has been shown to enter cells by utilizing both dynamin-dependent and -independent endocytic pathways. Thus, it is a representative ligand for addressing the important issue of whether even a relatively small ligand-nanoparticle conjugate can gain access to the same endocytic pathways as the free ligand.

Results: Here we present a systematic study concerning the internalization mechanism of ricinB:Quantum dot (QD) nanoparticle conjugates in HeLa cells. Contrary to uptake of ricin itself, we found that internalization of ricinB:QDs was inhibited in HeLa cells expressing dominant-negative dynamin. Both clathrin-, Rho-dependent uptake as well as a specific form of macropinocytosis involve dynamin. However, the ricinB:QD uptake was not affected by siRNAmediated knockdown of clathrin or inhibition of Rho-dependent uptake caused by treating cells with the Clostridium C3 transferase. RicinB:QD uptake was significantly reduced by cholesterol depletion with methyl- $\beta$ cyclodextrin and by inhibitors of actin polymerization such as cytochalasin D. Finally, we found that uptake of ricinB: QDs was blocked by the amiloride analog EIPA, an inhibitor of macropinocytosis. Upon entry, the ricinB:QDs colocalized with dextran, a marker for fluid-phase uptake. Thus, internalization of ricinB:QDs in HeLa cells critically relies on a dynamin-dependent macropinocytosis-like mechanism.
\end{abstract}

Conclusions: Our results demonstrate that internalization of a ligand-nanoparticle conjugate can be dependent on other endocytic mechanisms than those used by the free ligand, highlighting the challenges of using ligandmediated targeting of nanoparticles-based drug delivery vehicles to cells of diseased tissues.

Keywords: Nanoparticles, Ligand binding, Diagnostic imaging, Endocytic mechanisms, Ricin, Dynamin

\section{Background}

Nanomedicine is an interdisciplinary field of research focusing on the development of nanoparticles (NPs) for clinical use in targeted drug delivery and diagnostic in vivo imaging. The goal will often be to increase the efficacy of drugs/ siRNAs at the target tissue and reduce the dose of drug into bystander tissue, and/or to develop NPs into diagnostic imaging agents specifically targeting tumors and diseased tissues. However, studies to fundamentally understand the mechanisms of cell-nanoparticle interactions are still lacking. Investigating whether the nanoparticles themselves might have adverse effects is also

\footnotetext{
* Correspondence: toregi@rr-research.no

'Centre for Cancer Biomedicine, Faculty Division Norwegian Radium Hospital, University of Oslo, Oslo, Norway

${ }^{2}$ Department of Biochemistry, Institute for Cancer Research, The Norwegian Radium Hospital, Oslo University Hospital, Montebello, Oslo 0379, Norway Full list of author information is available at the end of the article
}

of crucial importance. The small sizes of nanoparticles enable them to cross various biological barriers of the body and also to enter the endocytic pathways of the cells, which in turn can give rise to unexpected toxicities. In a previous study, we demonstrated that cellular uptake of quantum dot (QD) nanocrystals that were surface modified with the targeting ligands transferrin (Tf) and ricin, perturbed normal intracellular trafficking in cells $[1,2]$.

There are multiple types of endocytic pathways distinguished by specific molecular regulators. The clathrin-mediated endocytosis is by far the best studied of these mechanisms and was for a long time believed to be the only endocytic mechanism in addition to phagocytosis and macropinocytosis. However, several clathrin-independent mechanisms have been described, including dynamin-dependent mechanisms such as the RhoA- and caveolae-dependent, and dynamin-independent 
mechanisms such as the Cdc42-dependent and Arf6dependent $[3,4]$. Dynamin is a large GTPase that mediates vesicle formation by its ability to tubulate and constrict membranes [5]. Caveolae-mediated uptake has been among the most studied routes of dynamin and cholesterol dependent endocytosis. In many studies uptake of nanoparticles (NPs) has been reported to occur via caveolae-mediated endocytosis merely based upon inhibited uptake by the pharmacological inhibitor methyl- $\beta$-cyclodextrin $(\mathrm{m} \beta \mathrm{CD})$. Notably, depleting the cell of cholesterol using $\mathrm{m} \beta \mathrm{CD}$ also inhibits other endocytic mechanisms, such as clathrinmediated endocytosis, phagocytosis and macropinocytosis [6,7]. Moreover, caveolae with a diameter of only $50-100 \mathrm{~nm}$ are clearly too small to be responsible for uptake of NPs larger than $100 \mathrm{~nm}$. Caveolae are present in most vascular endothelia playing an important role in transcytosis of blood-borne molecules across the vascular endothelial cell layer, and transcytosis of 10-15 nm gold NPs linked with a caveolaetargeting ligand has been shown [8]. The belief that internalization via caveolae would spare its cargo from being degraded in lysosomes has also been a reason for 'targeting' NPs to caveolae. However, the previous model of caveolae giving rise to neutral "caveosomes" has now been revised: The caveosomes are artefacts obtained by overexpression of caveolin-1, and a ligand taken up by caveolae will enter endosomes and be transported to lysosomes [9].

Although, macropinocytosis in general has been considered to be a dynamin-independent mechanism, the 'circular dorsal ruffle'-type of macropinocytosis might involve dynamin [10]. Macropinocytosis can in addition to fluid-phase uptake also accommodate uptake of particulate matter such as viruses, bacteria and nanoparticles $[11,12]$. Interestingly, dynamin-dependent and amiloridesensitive macropinocytosis-like mechanisms have been reported for the uptake of bluetongue virus-1 and the Ebola virus $[13,14]$. In endothelial cells, multimeric antibodynanoparticle conjugates directed against the intercellular adhesion molecule (ICAM-1) trigger internalization of large (diameter, 100-400 nm) anti-ICAM-1 and anti-PECAM-1 nanoconjugates by a macropinocytosislike mechanism that is dynamin-dependent and also requires RhoA activation and actin reorganization $[15,16]$. In a recent study, it has been shown that endocytosis of chemokines in endothelial cell lines occurred via a macropinocytosis-like process that was not blocked by siRNA knock-down of PAK1 and CtBP1, two effector proteins of the Rho family GTPase Rac1 [17]. Furthermore, it has been found that specific splice-variants of dynamin-2 were required for the internalization of fluid by endocytic pathways distinct from macropinocytosis [18].
Toxins such as the plant toxin ricin have for many years been used as valuable tools to study intracellular transport routes in cells, and also uptake of ricin coupled to small gold NPs has been shown in Vero cells [19-22]. Ricin consists of two polypeptide moieties linked by a disulfide bond. The B-moiety binds to glycolipids and glycoproteins with terminal galactose, and can therefore be used as a membrane marker. Studies of ricin endocytosis after inhibition of clathrin-dependent endocytosis by different methods demonstrated that ricin was still endocytosed, and these studies provided some of the first evidence for clathrin-independent endocytosis [23,24]. Moreover, ricin was still endocytosed after overexpression of the dominant negative mutant dynamin (dyn K44A/G273D) [25], which inhibited clathrin-mediated endocytosis of both transferrin (Tf) and epidermal growth factor (EGF), whereas fluid phase uptake of horse raddish peroxidase was unaffected [26]. Thus, ricin can be internalized by clathrin and dynamin independent mechanisms in HeLa cells. These endocytic mechanisms still remain incompletely characterized, but recently some of the proteins involved have been identified (for review see [3]). In a previous study, we found the internalized ricin:QD NPs localizing to the same early and late endosomes as ricin itself, but in contrast to ricin which is also transported to the Golgi apparatus, Golgi transport of the ricin:QD conjugate could not be observed [1]. Recently, a few other studies also revealed a change in ligand behavior after conjugation to NPs: It has been demonstrated in pancreatic cancer cell lines that anti-EGFR antibody-gold nanoparticle conjugates used different and faster endocytosis mechanisms than the anti-EGFR antibody itself [27], and the valency of TatP domains conjugated to QDs affected the fate of the NPs [28]. Furthermore, multivalent binding of antibodies and ligands of the PECAM-1 glycoprotein to NPs has been shown to trigger internalization of the antibody-ligand nanoconjugates in endothelial cells although the antibodies themselves were not internalized [29].

In this study we investigated by which endocytic mechanisms small $(30 \mathrm{~nm})$ ricinB:QD NP conjugates were internalized, and identified a dynamin-dependent macropinocytosis-like mechanism to be critically involved.

\section{Results and discussion}

Here, we have investigated the endocytic mechanisms responsible for internalization of ricinB:QDs, consisting of PEGylated streptavidin-coupled QDs that were conjugated with biotinylated ricinB (multivalent, molar ratio ricinB/QDs of 5), in HeLa cells. The size (hydrodynamic diam.) of the ricinB:QDs conjugates were measured by the Zetasizer to be $30 \mathrm{~nm}(5 \mathrm{~nm}$ variation between 
batches), small enough to be endocytosed by most endocytic mechanisms.

\section{Internalization of ricinB:QDs is mediated by dynamin- dependent endocytosis that is independent of clathrin} In our study of the endocytic mechanisms involved in uptake of ricinB:QD NP conjugates, we first investigated whether dynamin played a role. The role of dynamindependent endocytosis in uptake of ricinB:QDs was investigated in a $\mathrm{HeLa}$ cell line that expresses the dominant-negative dynamin-1 K44A mutant in a tetracycline-inducible manner [30]. Expression of the mutant dynamin K44A was induced by removal of tetracycline (-tetracycline) from the cells for $48 \mathrm{~h}$ prior to ricinB:QDs being added. Uptake of the fluorescent ricinB: QDs was analyzed by confocal microscopy (Figure 1). In control cells (A, +tetracycline), the ricinB:QDs were efficiently internalized within $20 \mathrm{~min}$ and found in endosomal 'dot-like' structures displaying a colocalization pattern with EEA1- and CD63-positive structures. In contrast, cells expressing the mutant dynamin $\mathrm{K} 44 \mathrm{~A}$ (B, -tetracycline) showed ricinB:QDs localized at the plasma membrane, and there was no significant colocalization with the endosomal markers. Furthermore, also during live-cell imaging of the HeLa cells, stained with the late endosomal (/low pH) marker LysoTracker, an inhibition in internalization of ricinB:QD and their colocalization with LysoTracker was observed (Figure 1B). However, after longer times of endocytosis, weak QD signals displaying colocalization with the endosomal markers (EEA1 and CD63) were also observed for the mutant dynamin expressing cells (-tet, $90 \mathrm{~min}$, lower panel). Colocalization of ricinB:QDs with the early endosomal marker EEA1 and the lysosomal marker CD63 was quantified after increasing time of endocytosis into the cells (Figure 1C). In HeLa cells expressing mutant dynamin, no significant internalization of ricinB:QDs (measured as fraction of cells with EEA1 colocalization) was detected until 45 min of incubation. Moreover, even after $90 \mathrm{~min}$ of endocytosis in the mutant dynamin cells, a reduced uptake and transport of ricinB:QDs into EEA1 and CD63-positive endosomes was quantified. In contrast, the majority of control cells displayed up to $50 \%$ colocalization with the lysosomal marker CD63. The binding of ricinB:QDs was not significantly affected by the expression of dynamin $\mathrm{K} 44 \mathrm{~A}$, and as expected the same cells displayed a strong inhibition of transferrin uptake due to a block in clathrin-mediated endocytosis (data not shown). Moreover, no significant binding or uptake of the unconjugated streptavidin-coated QDs was detected by confocal microscopy even after $4 \mathrm{~h}$ of incubation (negative control, not shown), confirming that conjugation of the QDs to biotinylated ricinB conferred specific binding and uptake into the cells. All together, our data show that dynamin is required for efficient uptake of the ricinB:QDs in HeLa cells. However, ricinB:QDs was also internalized slowly and inefficiently via a dynamin-independent mechanism.

In earlier studies, it has been shown that overexpression of mutant dynamin induced other compensatory dynamin-independent endocytic mechanisms that acted with similar kinetics in uptake of cargo such as ricin and the fluid phase marker HRP [25,31]. However, the ricinB:QDs can obviously not be internalized efficiently via this compensatory mechanism.

The next step was to examine by which dynamindependent mechanism the ricinB:QD bioconjugate is endocytosed. First, we investigated the uptake in HeLa cells expressing vector-based clathrin siRNA (Figure 2). The transfected cells expressing clathrin siRNA did not stain positive for clathrin, and in these cells the uptake of fluorescently labeled transferrin was strongly inhibited (cells outlined in white). However, these clathrin siRNA cells did not display any significant reduction in uptake of the ricinB:QDs as compared to the untransfected cells. In control cells transfected with a plasmid expressing a scramble siRNA, no cells without clathrin expression and inhibited internalization of Tf were observed (data not shown). Moreover, a BHK cell line with inducible expression of clathrin antisense RNA [32] also showed normal uptake of ricinB:Qdot nanoconjugates (data not shown).

\section{Cholesterol-dependent internalization of RicinB:QDs is independent of RhoA}

Because endocytosis of ricinB:QDs was critically dynamindependent, we further addressed whether an endocytic mechanism regulated by the small Rho-GTPases RhoA was involved [33]. The small GTPase RhoA also regulates formation of actin stress fibers and may be transiently activated during macropinocytosis [34]. To this end, we treated HeLa cells with the cell permeable C3 transferase, which selectively inhibits the small RhoGTPases RhoA-B and -C [35]. HeLa Tet dynK44A cells were pre-treated with $\mathrm{C} 3$ transferase for $90 \mathrm{~min}$ or left untreated. Then, the cells were allowed to internalize the ricinB:QDs for $40 \mathrm{~min}$ before they were fixed for IF microscopy. The uptake of ricinB:QDs was shown by confocal imaging as they colocalized with endosomal markers and could be visualized inside the cells by z-stack imaging (Figure 3). Most of the ricinB: QDs was endocytosed within $40 \mathrm{~min}$ both in control cells and in the cells treated with C3 transferase, indicating that RhoA-dependent endocytic mechanisms did not play any significant role. Usually, inhibition of Rho-dependent endocytosis with the C3 transferase coincides with a change in morphology where the cells get smaller and less 'spread out'. Here, we also observed 


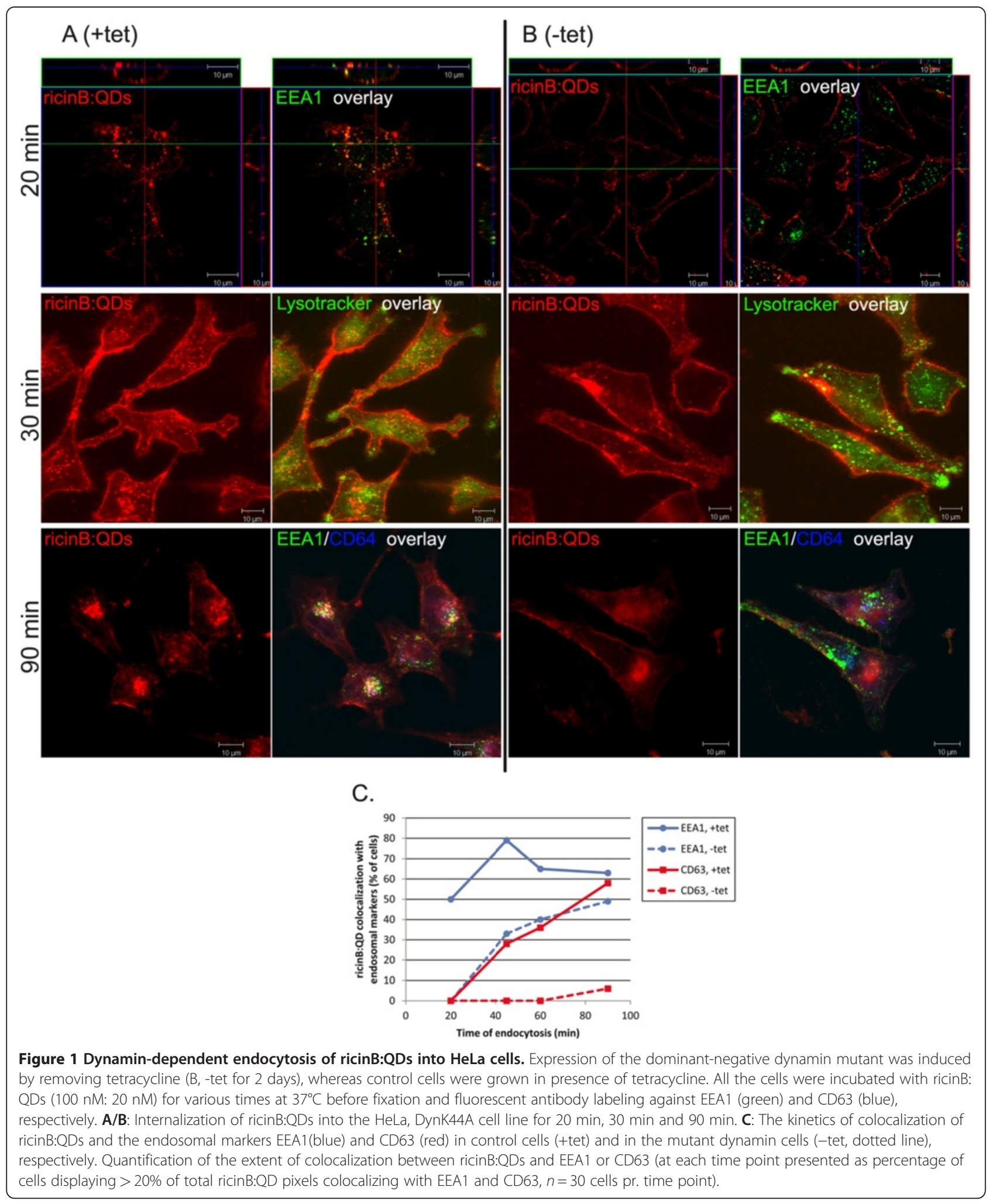

a similar change in morphology of the treated cells (Figure 3B). Furthermore, in a recent study we found that the C3 transferase inhibited internalization of the C. botulinum C2 toxin in these HeLa cells [36].
Cholesterol is a critical constituent of lipid rafts and has been reported to play an important role in various mechanisms of endocytosis such as clathrin-mediated, caveolaemediated, flotillin-mediated and macropinocytosis [3,7]. 

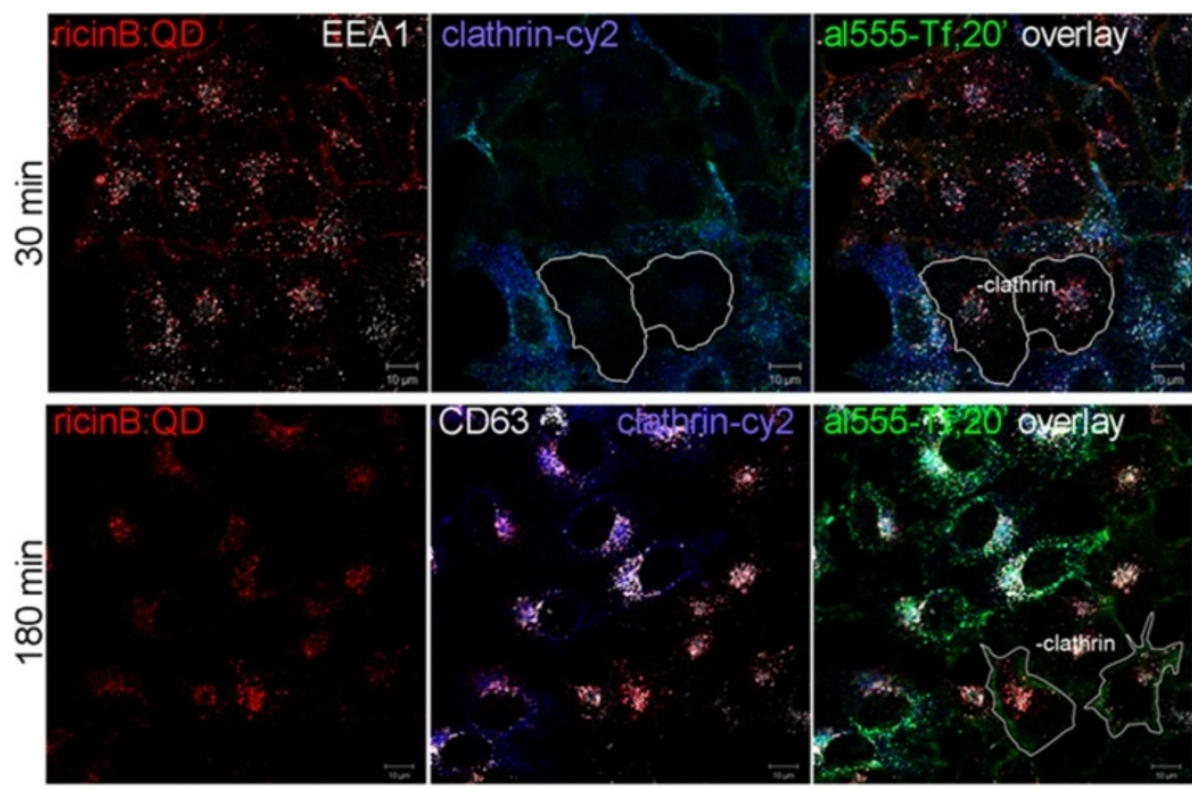

Figure 2 Clathrin independent entry of the ricinB:QDs into HeLa cells. HeLa cells were subjected to vector-based siRNA knock-down of clathrin heavy chain for 3 days. The ricinB:QD655 bioconjugates were allowed to be internalized into the cells at $37^{\circ} \mathrm{C}$ for $30 \mathrm{~min}$ and $180 \mathrm{~min}$, respectively (representative images, upper/lower panel). Alexa555 (al555)-transferrin (Tf) (green) was added to cells for the last 20 min of the endocytosis period. The cells were then fixed and prepared for confocal immuno-fluorescence microscopy labeling them with antibodies against clathrin heavy-chain, EEA1 and CD63, and with the appropriate secondary antibody-Cy2 (blue)/Cy5 (white) fluorophore-conjugates. Images show normal uptake of ricinB:QD655 in cells were clathrin is knocked down (- clathrin, no blue staining), whereas the alexa555-Tf uptake was inhibited and only weakly stained the periphery of these cells.

Therefore, we decided to deplete the HeLa cells for cholesterol by pretreatment for 30 min with the inhibitor $\mathrm{m} \beta \mathrm{CD}$ $(10 \mu \mathrm{M})$. The cells were then allowed to internalize ricinB: QDs (Figure 4A,B) or ricinB-biotin:streptavidin-Cy3 conjugates together with Tf (Figure 4C), respectively for $30 \mathrm{~min}$. Figure $4 \mathrm{~B}$ shows that the uptake of ricinB:QDs was strongly reduced in cells depleted of cholesterol, since no colocalization with the endosomal marker EEA1 was observed and side views of the cells revealed ricinB:QD staining of the cell surface only. This result shows the involvement of cholesterol in uptake of the ricinB:QDs. Notably, caveolae-mediated endocytosis is not likely to play any important role in the uptake of ricinB:QDs, because the HeLa dynK44A cells contain very few caveolae [37]. In
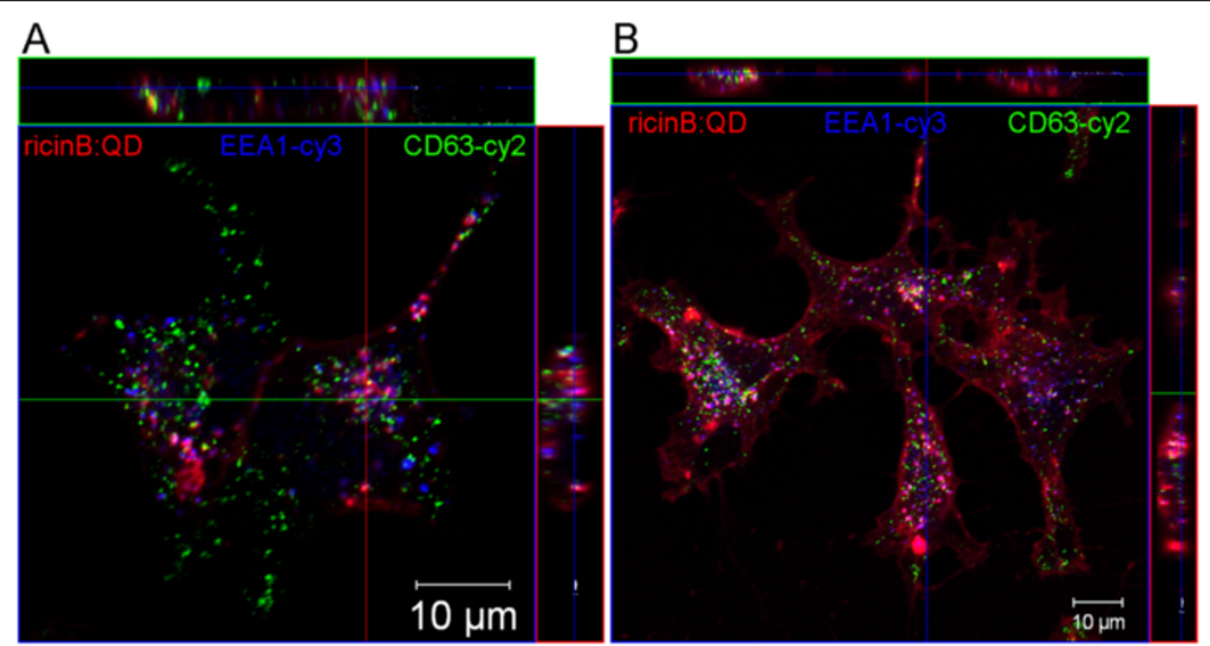

Figure 3 Uptake of ricinB:QDs is not reduced by inhibition of Rho. (A) Cells were left untreated, or (B) pre-treated with C3 transferase (1 $\mu \mathrm{g} / \mathrm{ml})$, followed by incubation with ricinB:QDs at $37^{\circ} \mathrm{C}$ for $40 \mathrm{~min}$. Then, the cells were fixed and stained with the appropriate antibodies against the endosomal marker EEA1 (blue) and CD63 (green), respectively. Images include orthogonal views generated from a z-stack of images. 


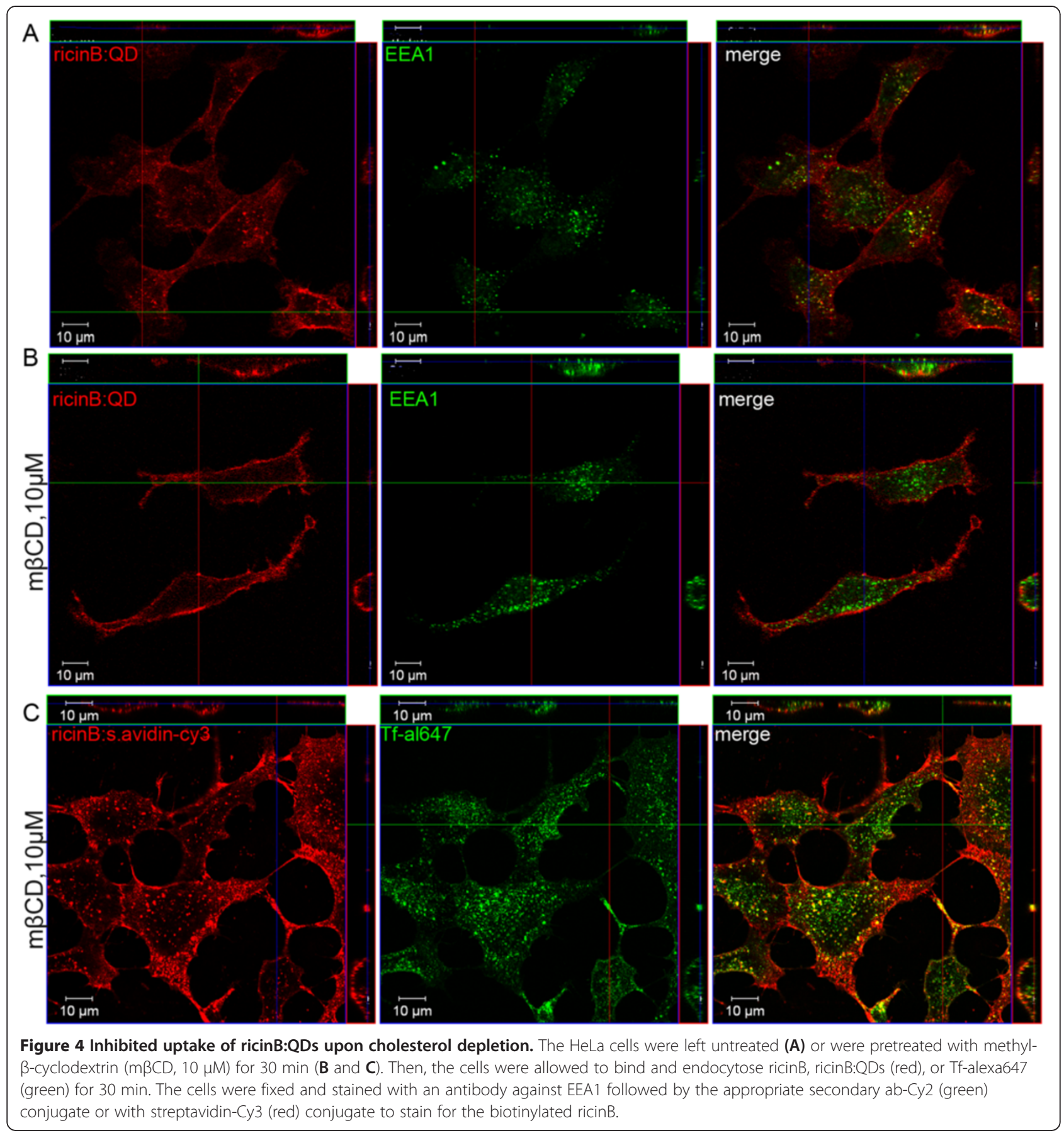

a previous study, we demonstrated that cholesterol depletion by $\mathrm{m} \beta C \mathrm{C}$ in HeLa cells only reduced endocytosis of Tf by $50 \%$ whereas ricin uptake was reduced by $<20 \%$ [6]. Thus, as a positive control we show that the cells treated with $\mathrm{m} \beta \mathrm{CD}$ still internalized ricinB and Tf (Figure $4 \mathrm{C}$ ), as fluorescently labeled ricinB (red color) and Tf (green color) displayed significant colocalization in endosomal 'dots' within the cells.
Effects of actin inhibitors on the ricinB:QD endocytosis In order to address the role of actin filament assembly in uptake of the ricinB:QDs, HeLa cells were pre-treated with the inhibitor cytochalasin $\mathrm{D}(\mathrm{cytD}, 5 \mu \mathrm{M})$. CytD is a cell-membrane permeant that caps actin filaments thereby preventing further polymerization and ultimately resulting in actin filament disassembly. As seen in Figure 5B, uptake of the ricinB:QDs was strongly reduced by cytD, 
A
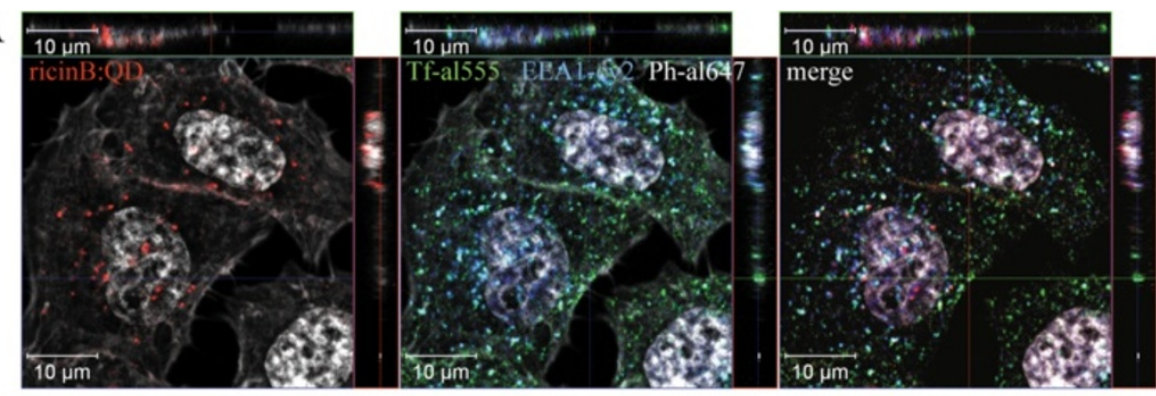

B
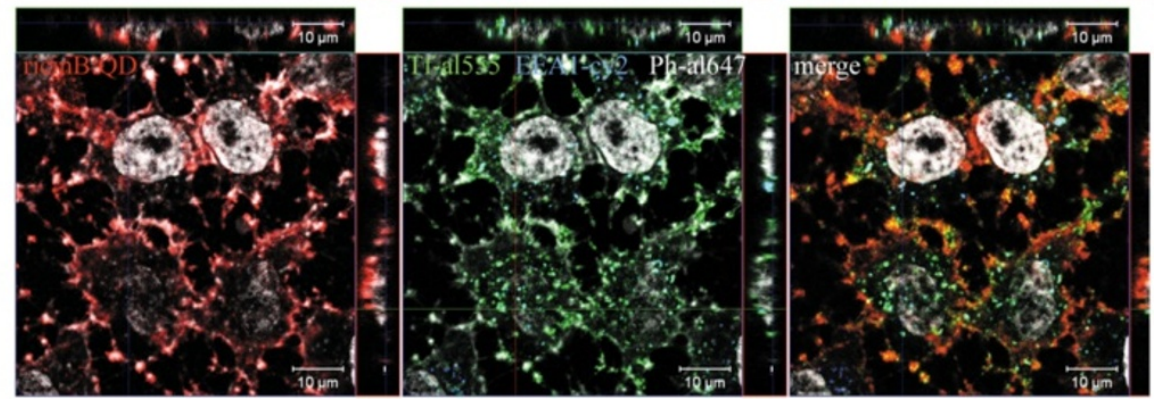

Figure 5 Actin-dependent uptake of the ricinB:QDs. HeLa cells were left untreated (A) or treated with cytD (B). Then, ricinB:QDs (in red) and transferrin- alexa555 (Tf-al555, green) were endocytosed for $30 \mathrm{~min}$ at $37^{\circ} \mathrm{C}$. The cells were fixed and stained with an anti-EEA 1 antibody and the appropriate secondary antibody-Cy2 conjugate (blue) together with phalloidin (Ph)-alexa647 (white) for staining of actin. The cell nuclei were stained with Hoechst33342 (white).

as most ricinB:QDs were localized along the cell periphery. Uptake of Tf-al555 was not significantly inhibited by cytD and displayed colocalization with EEA1 (blue) in endosomal 'spots'.

In contrast to our findings, endocytosis of small particles such as QDs $(<100 \mathrm{~nm})$ have been reported to be insensitive to cytD in mammalian macrophages, leukocytes and dendritic cells [38,39]. However, disruption of $\mathrm{F}$ actin by cytD has been reported to play a variable role in endocytosis of Tf depending on both the cell line and whether the cells were grown in suspension or not $[40,41]$. Recently, is has been shown that membrane tension can decide whether actin is required for clathrindependent endocytosis [41].

\section{Internalization of ricinB:QDs is mediated by a macropinocytosis-like mechanism}

Macropinocytosis is known to be critically dependent on the actin cytoskeleton for generating the plasma membrane 'ruffles' or filopodia that fold back on the plasma membrane to form the macropinosomes. Macropinocytosis is dependent upon the amiloride-sensitive $\mathrm{Na}^{+} / \mathrm{H}^{+}$ exchanger in the plasma membrane and is inhibited by 5-(N-ethyl-N-isopropyl)-amiloride (EIPA). In a recent study it has been shown that the inhibitory effects of amiloride and related compounds are the consequence of submembranous acidification caused by $\mathrm{H}^{+}$generation and the blocked $\mathrm{Na}^{+} / \mathrm{H}^{+}$exchange across the plasma membrane [42]. The macropinosomes that pinches off from the cell surface can be identified through the use of fluid phase markers such as dextran, horse radish peroxidase and Lucifer Yellow. Upon cointernalization of ricinB:QDs and dextran, we observed good co-localization between them within endosomal structures (yellow color) even after short times of internalization ( $<30 \mathrm{~min}$., Figure $6 \mathrm{~A}$ ), indicating that they were endocytosed by the same mechanism. To ascertain that this colocalization was not an artifact due to cross-talk of the QD signal into the dextran-alexa594. channel, we showed that the dextran signal within the rectangle area (Figure 6A, inset shows pre-bleech) could be photo-bleached using high laser power whereas the QD signal persisted. Furthermore, we observed an increasing colocalization between ricinB:QDs and the lysosomal marker CD63 from $30 \mathrm{~min}$ to $90 \mathrm{~min}$ (shown as purple color, Figure $6 \mathrm{~A}, \mathrm{C}$ insets), indicative of routing of the ricinB:QDs into lysosomes/late endosomes. Importantly, Figure 6 (B and D) shows that EIPA efficiently inhibited uptake of the ricinB:QDs and dextran in HeLa cells. In the EIPA-treated cells, the ricinB: QDs were still retained at the cell surface after $90 \mathrm{~min}$ of endocytosis. In contrast, EIPA did not inhibit uptake of transferrin as most EIPA-treated cells showed Tf uptake (not shown).

The activation of PI3K and the engagement of signaling molecules including Rac1, Arf6 and the RhoA GTPase are common to a variety of actin-dependent 


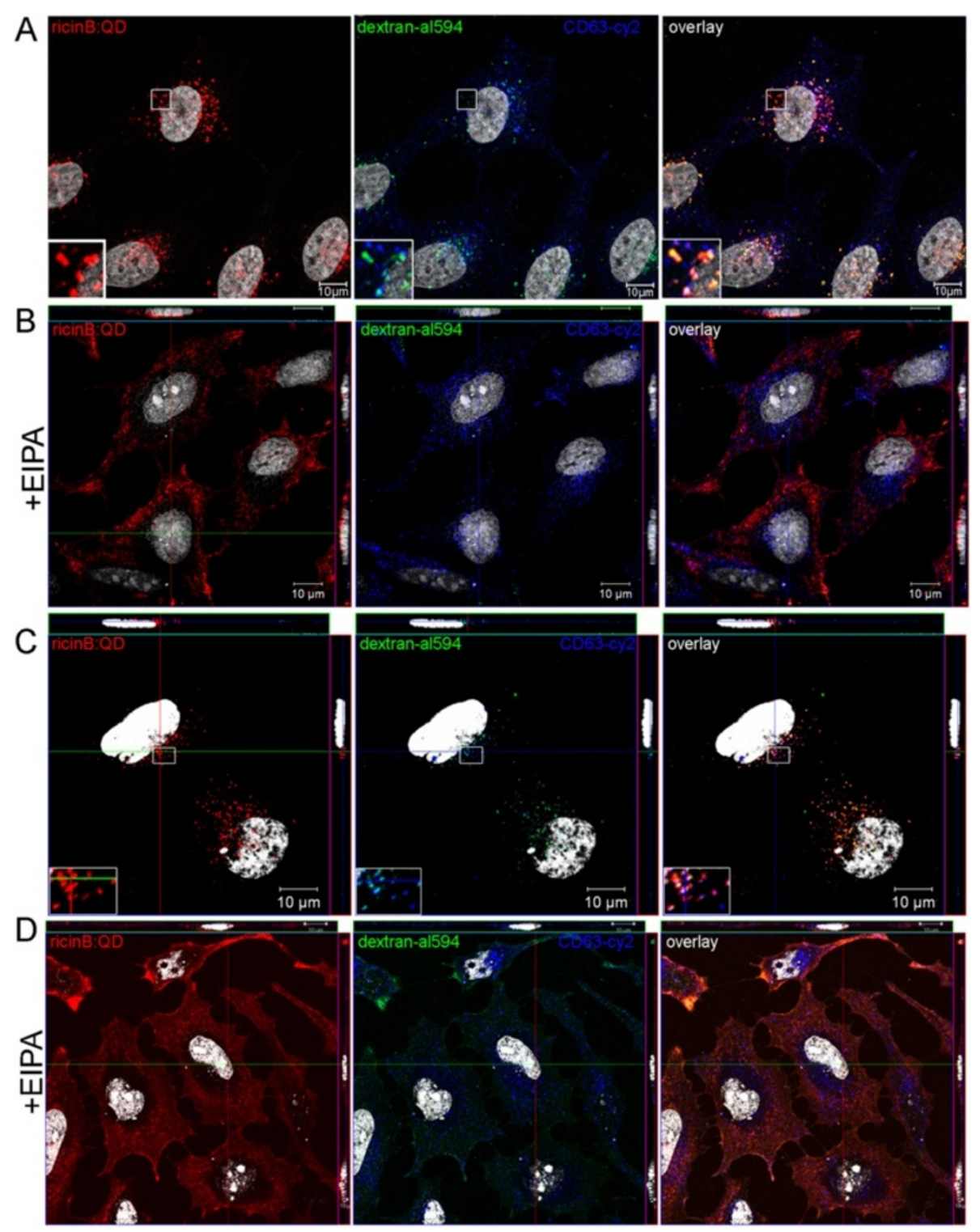

Figure 6 Inhibition of ricinB:QDot endocytosis upon treatment of cells with EIPA, an inhibitor of macropinocytosis. RicinB: QDs (red) were pre-bound to HeLa cells at $4^{\circ} \mathrm{C}$, and then internalized in the presence of alexa594 dextran (green) for 30 min (panel A,B) and 90 min (panel C,D), respectively. The cells were fixed and stained with lysosomal antibody CD63 and its corresponding secondary antibody-Cy2 (blue). The cell nuclei were stained with Hoechst33342 (white).

processes such as phagocytosis and macropinocytosis [43]. In this context, it is interesting to note that in this study uptake of ricinB: QDs were not inhibited by treatment of the HeLa cells with the inhibitor of Rho, C3 transferase (see Figure 3). Furthermore, overexpression of the dominant-negative mutant Arf6 T27N in a stably transfected HeLa cell line [44] did not inhibit endocytosis of ricinB: QDs (data not shown). Macropinocytosis has been considered as a regulated form of endocytosis induced in response to growth factors such as epidermal growth factor [45]. Therefore, we would like to know whether the ricinB: QDs could induce such a fluid phase pathway. Using the Olympus ScanR fluorescence microscope, we quantified the uptake of fluorescent dextran-alexa594 in HeLa cells when taken up alone or when co-internalized with the ricinB:QDs for $30 \mathrm{~min}$. No differences in the fluorescence intensities of internalized dextran were measured between the two conditions (data 
not shown), indicating that uptake of the ricinB:QDs did not significantly change the fluid uptake of dextran during this time period.

\section{Conclusions}

In this study we investigated the relevance of various endocytic pathways in the uptake of ricin-coupled QD nanoparticles conjugates in HeLa cells. We demonstrated that the ricin-QDs conjugates were endocytosed by a dynamin-dependent but clathrin-independent pathway. Furthermore, treatment of the cells with C3 transferase to inhibit the Rho-mediated endocytic mechanism did not affect ricinB:QD uptake. Depletion of cholesterol from the cells, significantly reduced the uptake of ricinB: QDs. Interestingly, treatment of the cells with an inhibitor of macropinocytosis, the amiloride-analog EIPA, strongly reduced the uptake of ricinB: QDs. Thus, we have demonstrated that a dynamin-dependent and macropinocytosis-like pathway is necessary for the efficient endocytosis of ricinB:QD nanoconjugates. In contrast, ricin alone is internalized equally efficiently both by dynamin-dependent and dynamin-independent endocytic pathways. These endocytic pathways seem to be unavailable for internalization of the ricinB:QDs, and their uptake is instead triggered via a specific endocytic pathway (Figure 7). These results demonstrate that one needs to be cautious when ligands are coupled to nanoparticles with the intention of achieving efficient NP delivery into certain cells or tissues by a given endocytic mechanism. Clearly, the endocytic pathways operating after nanoconjugation warrants further investigations. The size of the NPs to which the ligands are conjugated is certainly also an issue, as larger NPs cannot enter into some endocytic pathways due to sterical hindrance. Importantly, the multivalency of ligand-nanoparticle conjugates might not only increase the affinity for a cell, but also enable more efficient receptor clustering resulting in induction of signaling and thereby triggering of alternative endocytic pathways.

\section{Methods}

\section{Experimental reagents}

Reagents used in this study included the amiloride analoge EIPA (Sigma), cytochalasin D (Sigma), methyl- $\beta$ cyclodextrin (Sigma), ADP-ribosyltranferase C3 (Sigma). RicinB subunit was purchased from Vector Laboratories (Burlingame, CA). Quantum dots $655 \mathrm{~nm}$, alexa-555 transferrin (Tf), alexa-594 dextran, alexa-647 phalloidin $(\mathrm{Ph})$, and Hoechst 33342 were purchased from Invitrogen (Carlsbad, CA). The following antibodies were used: Rabbit anti-EEA1 (Cell Signaling Technologies), mouse anti-CD63 (Developmental Studies Hybridoma Bank, Univ. of Iowa). The Cy2- and Cy3-labeled secondary antibody conjugates of donkey anti-rabbit and donkey anti-mouse were purchased from Jackson ImmunoResearch Laboratories (West Grove, PA, USA).

\section{Biotinylation of ricinB}

RicinB (1 $\mathrm{mg} / \mathrm{ml})$ was biotinylated according to manufacturer's instructions using NHS-SS-biotin (Pierce, Rockford, IL) in a 1:20 molar ratio of ricin:biotin. The reaction took place at room temperature in the dark for $45 \mathrm{~min}$. Unincorporated NHS-SS-biotin was removed using a spin column (Micron, YM-10, Millipore).

\section{Hydrodynamic size measurement}

The hydrodynamic diameter of the streptavidin-coupled QD655 particles was measured in PBS buffer or in cell culture medium using using a Zetasizer Nano ZS (Malvern Instruments Ltd, Worcestershire, UK).

\section{Cell culture}

$\mathrm{HeLa}$ cells were maintained at $37^{\circ} \mathrm{C}$ and $5 \% \mathrm{CO}_{2}$ in Dulbecco's Modified Eagle medium, DMEM (Invitrogen,

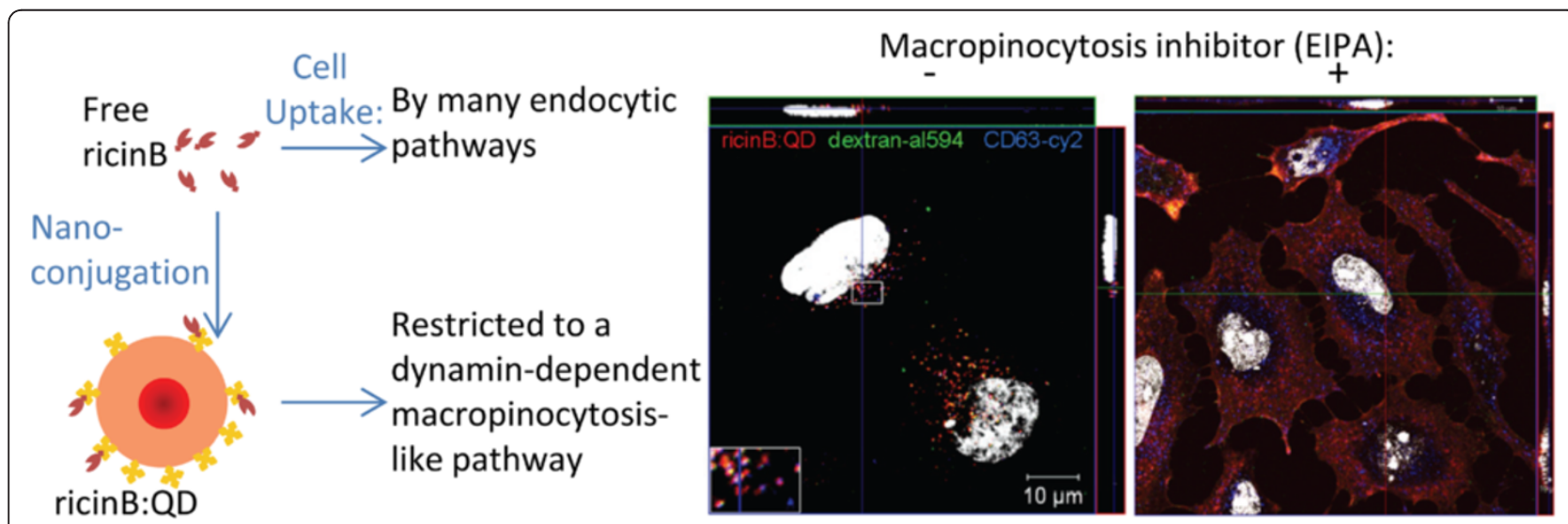

Figure 7 Endocytosis of ricinB:QD nanocomplexes is restricted to a macropinocytosis-like pathway. Ricin:QD NPS cannot exploit the various endocytic pathways accessible to ricin toxin itself. 
Carlsbad, CA, USA), supplemented with $10 \% \mathrm{v} / \mathrm{v}$ fetal bovine serum (PAA Laboratories, Linz, Austria), $100 \mathrm{U} / \mathrm{ml}$ penicillin (Invitrogen) and $100 \mu \mathrm{g} / \mathrm{ml}$ streptomycin (Invitrogen).

\section{Cellular uptake of ricinB:qdot nanoconjugates by confocal fluorescence microscopy}

HeLa cells or HeLa Dyn-K44A cells (seeded $2 \times 10^{4}$ cells/ well in 24-well trays) were cultured on coverslips for $24 \mathrm{~h}$ or $48 \mathrm{~h}$ prior to the experiments, respectively. Then, ricinB:QDs conjugates were prepared directly at the cell surface, as previously described:[1] Cells were washed in cold Hepes-medium prior to incubation with the biotinylated ricinB $(200 \mathrm{nM})$ ligand in Hepes medium for $10 \mathrm{~min}$ on ice. The cells were briefly washed $2 \mathrm{x}$ in tetra-borate buffer $(50 \mathrm{mM}$ sodium-borate, $\mathrm{pH} 8.3$; $215 \mathrm{mM}$ sucrose) and then incubated with the streptavidin-coupled QD655 (20 $\mathrm{nM})$ in tetra-borate buffer for $5 \mathrm{~min}$ on ice [46]. Subsequently, the cells were washed $2 \mathrm{x}$ in Hepes medium before endocytosis of the ricinB:QDs (molar ratio, 5:1) was performed by incubating the cells in Hepes medium at $37 \mathrm{C}$ for various times with or without the appropriate inhibitors. The ricinB: QDs were also co-internalized with other ligands such as Tf-alexa-555 and dextran-alexa-647.

After fixation in 10\% (w/v) formalin for $15 \mathrm{~min}$, the cells were permeabilized and blocked in $0.1 \%$ Triton $\mathrm{X}-100$ and $1 \%$ BSA in PBS for 1 hour at room temperature. The cells were immuno-stained with the following primary antibodies: mouse anti-EEA1 ab. $(1: 100)$ and mouse anti-CD63 ab. (1:200). The secondary antibody-fluorescent dye conjugates used were: Donkey anti-rabbit-Cy2 (1:200), donkey anti-rabbitCy3 (1:500) and donkey anti-mouse-Cy2 (1:500). Coverslips were mounted in Mowiol (Calbiochem) and examined using a confocal microscope (LSM 788; Carl Zeiss MicroImaging, Inc.) equipped with a Neo-Fluar $63 x / 1.45$ oil immersion objective. Image processing and analysis were done with Zeiss LSM 510 software version 3.2 and Adobe Photoshop 7.0.

\section{Vector-based siRNA knock-down of clathrin}

Clathrin heavy-chain was knocked down with a vectorbased siRNA construct [47]. Cells were transiently transfected for 3 days with plasmid DNA using Fugene 6 (Roche Diagnostics, Mannheim, Germany), according to the manufacturer's protocol.

\section{Competing interests}

The authors declare that they have no competing interests.

\section{Authors' contributions}

TGl conceived the idea of this study and participated in its design, carried out most of the experiments on endocytosis mechanisms and drafted the manuscript. NF carried out quantitative colocalization analysis of confocal images using the Zeiss LSM 510 software (version 3.2), and performed the cellular uptake study after cholesterol depletion using methyl- $\beta$-cyclodextrin. KS participated in study design and coordination and helped draft the manuscript. All authors read and approved the final manuscript.

\section{Acknowledgements}

Tore-Geir Iversen and Nadine Frerker were the recipients of career and postdoctoral fellowships, respectively, from the FUGE and NANOMAT programmes. This work was supported by the Research Council of Norway, the Norwegian Cancer society and Helse Sør-Øst.

\section{Author details}

${ }^{1}$ Centre for Cancer Biomedicine, Faculty Division Norwegian Radium Hospital, University of Oslo, Oslo, Norway. ${ }^{2}$ Department of Biochemistry, Institute for Cancer Research, The Norwegian Radium Hospital, Oslo University Hospital, Montebello, Oslo 0379, Norway. ${ }^{3}$ Department of Molecular Biosciences, University of Oslo, Oslo 0316, Norway.

Received: 18 May 2012 Accepted: 23 July 2012

Published: 31 July 2012

\section{References}

1. Tekle C, van Deurs B, Sandvig K, Iversen TG: Cellular Trafficking of Quantum Dot-Ligand Bioconjugates and Their Induction of Changes in Normal Routing of Unconjugated Ligands. Nano Letters 2008, 8:1858-1865.

2. Iversen TG, Frerker N, Sandvig K: Quantum dot bioconjugates: uptake into cells and induction of changes in normal cellular transport. San Jose, CA, USA: SPIE: Progress in biomedical optics and imaging; 2009:71890T-9.

3. Sandvig K, Pust S, Skotland T, van DB: Clathrin-independent endocytosis: mechanisms and function. Curr Opin Cell Biol 2011, 23:413-420.

4. Iversen TG, Skotland T, Sandvig K: Endocytosis and intracellular transport of nanoparticles: Present knowledge and need for future studies. Nano Today 2011, 6:176-185

5. Doherty GJ, McMahon HT: Mechanisms of endocytosis. Annu Rev Biochem 2009, 78:857-902.

6. Rodal SK, Skretting G, Garred O, Vilhardt F, van Deurs B, Sandvig K. Extraction of cholesterol with methyl-beta-cyclodextrin perturbs formation of clathrin-coated endocytic vesicles. Mol Biol Cell 1999, 10:961-974

7. Grimmer S, van Deurs B, Sandvig K: Membrane ruffling and macropinocytosis in A431 cells require cholesterol. J Cell Sci 2002, 115:2953-2962.

8. Mclntosh DP, Tan XY, Oh P, Schnitzer JE: Targeting endothelium and its dynamic caveolae for tissue-specific transcytosis in vivo: a pathway to overcome cell barriers to drug and gene delivery. Proc Natl Acad Sci U S A 2002, 99:1996-2001.

9. Hayer A, Stoeber M, Ritz D, Engel S, Meyer HH, Helenius A: Caveolin-1 is ubiquitinated and targeted to intralumenal vesicles in endolysosomes for degradation. J Cell Biol 2010, 191:615-629.

10. Orth JD, McNiven MA: Get off my back! Rapid receptor internalization through circular dorsal ruffles. Cancer Res 2006, 66:11094-11096.

11. Mercer J, Helenius A: Apoptotic mimicry: phosphatidylserine-mediated macropinocytosis of vaccinia virus. Ann N Y Acad Sci 2010, 1209:49-55.

12. Lim JP, Gleeson PA: Macropinocytosis: an endocytic pathway for internalising large gulps. Immunol Cell Biol 2011, 89:836-843.

13. Gold S, Monaghan P, Mertens $\mathrm{P}$, Jackson T: A clathrin independent macropinocytosis-like entry mechanism used by bluetongue virus-1 during infection of BHK cells. PLoS One 2010, 5:e11360.

14. Mulherkar N, Raaben M, de la Torre JC, Whelan SP, Chandran K: The Ebola virus glycoprotein mediates entry via a non-classical dynamindependent macropinocytic pathway. Virology 2011, 419:72-83.

15. Muro S, Wiewrodt R, Thomas A, Koniaris L, Albelda SM, Muzykantov VR, et al: A novel endocytic pathway induced by clustering endothelial ICAM-1 or PECAM-1. J Cell Sci 2003, 116:1599-1609.

16. Garnacho C, Shuvaev V Thomas A, McKenna L, Sun J, Koval M, et al: RhoA activation and actin reorganization involved in endothelial CAMmediated endocytosis of anti-PECAM carriers: critical role for tyrosine 686 in the cytoplasmic tail of PECAM-1. Blood 2008, 111:3024-3033.

17. Zhao Y, Mangalmurti NS, Xiong Z, Prakash B, Guo F, Stolz DB, et al: Duffy Antigen Receptor for Chemokines Mediates Chemokine Endocytosis 
through a Macropinocytosis-Like Process in Endothelial Cells. PLoS One 2011, 6:e29624.

18. Cao H, Chen J, Awoniyi M, Henley JR, McNiven MA: Dynamin 2 mediates fluid-phase micropinocytosis in epithelial cells. J Cell Sci 2007, 120:4167-4177.

19. Sandvig K, Torgersen ML, Engedal N, Skotland T, Iversen TG: Protein toxins from plants and bacteria: probes for intracellular transport and tools in medicine. FEBS Lett 2010, 584:2626-2634.

20. Sandvig K, Spilsberg B, Lauvrak SU, Torgersen ML, Iversen TG, van Deurs B: Pathways followed by protein toxins into cells. Int J Med Microbiol 2004, 293:483-490.

21. van Deurs B, Hansen SH, Petersen OW, Melby EL, Sandvig K: Endocytosis, intracellular transport and transcytosis of the toxic protein ricin by a polarized epithelium. Eur J Cell Biol 1990, 51:96-109.

22. van Deurs B, Pedersen LR, Sundan A, Olsnes S, Sandvig K: Receptormediated endocytosis of a ricin-colloidal gold conjugate in Vero cells Intracellular routing to vacuolar and tubulo-vesicular portions of the endosomal system. Exp Cell Res 1985, 159:287-304.

23. Sandvig K, Olsnes S, Petersen OW, van Deurs B: Acidification of the cytosol inhibits endocytosis from coated pits. J Cell Biol 1987, 105:679-689.

24. Moya M, Dautry-Varsat A, Goud B, Louvard D, Boquet P: Inhibition of coated pit formation in Hep2 cells blocks the cytotoxicity of diphtheria toxin but not that of ricin toxin. J Cell Biol 1985, 101:548-559.

25. Llorente A, Rapak A, Schmid SL, van Deurs B, Sandvig K: Expression of Mutant Dynamin Inhibits Toxicity and Transport of Endocytosed Ricin to the Golgi Apparatus. J Cell Biol 1998, 140:553-563.

26. Damke $H$, Baba T, Warnock DE, Schmid SL: Induction of mutant dynamin specifically blocks endocytic coated vesicle formation. J Cell Biol 1994, 127:915-934.

27. Bhattacharyya S, Bhattacharya R, Curley S, McNiven MA, Mukherjee P: Nanoconjugation modulates the trafficking and mechanism of antibody induced receptor endocytosis. Proc Natl Acad Sci U S A 2010, 107:14541-14546.

28. Imamura J, Suzuki Y, Gonda K, Roy CN, Gatanaga H, Ohuchi N, et al: Single particle tracking confirms that multivalent Tat protein transduction domain-induced heparan sulfate proteoglycan cross-linkage activates Rac1 for internalization. J Biol Chem 2011, 286:10581-10592.

29. Muzykantov VR, Christofidou-Solomidou M, Balyasnikova I, Harshaw DW Schultz $L$, Fisher $A B$, et al: Streptavidin facilitates internalization and pulmonary targeting of an anti-endothelial cell antibody (plateletendothelial cell adhesion molecule 1): a strategy for vascular immunotargeting of drugs. Proc Natl Acad Sci U S A 1999, 96:2379-2384.

30. Damke H, Gossen M, Freundlieb S, Bujard H, Schmid SL: Tightly regulated and inducible expression of dominant interfering dynamin mutant in stably transformed HeLa cells. Methods Enzymol 1995, 257:209-220.

31. Damke H, Baba T, van der Bliek AM, Schmid SL: Clathrin-independent pinocytosis is induced in cells overexpressing a temperature-sensitive mutant of dynamin. J Cell Biol 1995, 131:69-80.

32. Iversen TG, Skretting G, van Deurs B, Sandvig K: Clathrin-coated pits with long, dynamin-wrapped necks upon expression of a clathrin antisense RNA. Proc Natl Acad Sci U S A 2003, 100:5175-5180.

33. Lamaze C, Dujeancourt A, Baba T, Lo CG, Benmerah A, utry-Varsat A: Interleukin 2 receptors and detergent-resistant membrane domains define a clathrin-independent endocytic pathway. Mol Cell 2001 7:661-671.

34. Mercer J, Knebel S, Schmidt FI, Crouse J, Burkard C, Helenius A: Vaccinia virus strains use distinct forms of macropinocytosis for host-cell entry. Proc Natl Acad Sci U S A 2010, 107:9346-9351.

35. Schmalzing G, Richter HP, Hansen A, Schwarz W, Just I, Aktories K. Involvement of the GTP binding protein Rho in constitutive endocytosis in Xenopus laevis oocytes. J Cell Biol 1995, 130:1319-1332

36. Pust $\mathrm{S}$, Barth $\mathrm{H}$, Sandvig $\mathrm{K}$ : Clostridium botulinum $\mathrm{C} 2$ toxin is internalized by clathrin- and Rho-dependent mechanisms. Cell Microbiol 2010, 12:1809-1820.

37. Torgersen ML, Skretting G, van Deurs B, Sandvig K: Internalization of cholera toxin by different endocytic mechanisms. J Cell Sci 2001, 114:3737-3747.

38. Brewer JM, Pollock KG, Tetley L, Russell DG: Vesicle size influences the trafficking, processing, and presentation of antigens in lipid vesicles. $\mathrm{J}$ Immunol 2004, 173:6143-6150.
39. Sen D, Deerinck TJ, Ellisman MH, Parker I, Cahalan MD: Quantum Dots for Tracking Dendritic Cells and Priming an Immune Response In Vitro and In Vivo. Plos One 2008, 3:e3290.

40. Fujimoto LM, Roth R, Heuser JE, Schmid SL: Actin assembly plays a variable, but not obligatory role in receptor-mediated endocytosis in mammalian cells. Traffic 2000, 1:161-171.

41. Boulant S, Kural C, Zeeh JC, Ubelmann F, Kirchhausen T: Actin dynamics counteract membrane tension during clathrin-mediated endocytosis. Nat Cell Biol 2011, 13:1124-1131.

42. Koivusalo M, Welch C, Hayashi H, Scott CC, Kim M, Alexander T, et al: Amiloride inhibits macropinocytosis by lowering submembranous $\mathrm{pH}$ and preventing Rac1 and Cdc42 signaling. J Cell Biol 2010, 188:547-563.

43. Donaldson JG, Porat-Shliom N, Cohen LA: Clathrin-independent endocytosis: a unique platform for cell signaling and PM remodeling. Cell Signal 2009, 21:1-6.

44. Raa H: Endosome-to-Golgi transport of ricin is influenced by ADP-ribosylation factor 6. University of Oslo: Thesis for Master Degree in Biochemistry, Department of Molecular Biosciences; 2006.

45. Racoosin EL, Swanson JA: Macrophage colony-stimulating factor (rM-CSF) stimulates pinocytosis in bone marrow-derived macrophages. J Exp Med 1989, 170:1635-1648.

46. Clapp AR, Medintz IL, Uyeda HT, Fisher BR, Goldman ER, Bawendi MG, et al: Quantum Dot-Based Multiplexed Fluorescence Resonance Energy Transfer. J Am Chem Soc 2005, 127:18212-18221.

47. Skanland SS, Walchli S, Brech A, Sandvig K: SNX4 in complex with clathrin and dynein: implications for endosome movement. PLoS One 2009, 4:e5935

doi:10.1186/1477-3155-10-33

Cite this article as: Iversen et al: Uptake of ricinB-quantum dot nanoparticles by a macropinocytosis-like mechanism. Journal of Nanobiotechnology 2012 10:33.

\section{Submit your next manuscript to BioMed Central and take full advantage of:}

- Convenient online submission

- Thorough peer review

- No space constraints or color figure charges

- Immediate publication on acceptance

- Inclusion in PubMed, CAS, Scopus and Google Scholar

- Research which is freely available for redistribution 